\title{
Robust Transmission of prioritized H.264/AVC employing linear block codes and non-uniform 8PSK modulation for wireless communications
}

\author{
Mussawir Ahmad Hosany ${ }^{1}$, Ridwaan Hosany ${ }^{2}$ \\ Department of Electrical and Electronic Engineering, Faculty of Engineering, University of Mauritius, \\ Mauritius
}

\begin{abstract}
Due to the fruitful and diversified contents of multimedia traffic and the vulnerable environment of wireless communications, applying unequal error protection (UEP) is now an active research topic. In this paper, we present a novel and alternative method of providing UEP by combining the channel code with the modulation-assisted symbol. We use the powerful BCH code and non-uniform $8 P S K$ modulation to handle UEP of the prioritized H.264/AVC bit stream. Based on the non-uniformly distributed property of the importance of intracoded (I-frame) and the predictive coded frame (p-frame), we have found a suitable approach for the nonuniform 8PSK signal constellation. Simulation results show that our proposed UEP scheme is able to significantly improve the peak signal-to-noise ratio (PSNR). It has been shown that at a value of $E_{b} / N_{o}$ of $14 d B$ the video sequence transmitted at a bit rate of 512 Kbps yields a 4 dB gain in PSNR compared with the uniform $8 P S K$ modulation case which makes the proposed UEP scheme robust to wireless communication errors.
\end{abstract}

Keywords: H.264/AVC, BCH code, Hierarchical modulation, wireless channels, Unequal error protection

\section{Introduction}

Recently there has been major focus on the H.264/AVC standard since it offers the best compression efficiency over its predecessor such as MPEG-4 [1] and makes proper use of the available channel bandwidth. Novel and robust transmission schemes for H.264/AVC video data have to be developed due to the increasing demand in multimedia services over wireless channels. Different types of error resiliency schemes are used in the H.264 video compression to reduce the effect of transmission. Examples of such schemes include data partitioning, layered coding, reference frame selection, Flexible Macroblock Ordering (FMO) in H.264/AVC video coding standard [2,3].

In order to minimize the effects of transmission errors on the reconstructed video image quality, Unequal Error Protection (UEP) schemes can be used [4-7]. UEP is based on the idea that the more important video data is given higher protection at the cost of the less important data. For example, the picture header and motion vectors are much more important than the texture data. The reconstructed video quality will be severely degraded when errors occur on the important bits. Thus, these important bits should have a higher protection order than the rest of the video bit-stream.

At the application layer in order to exploit unequal error protection of the video bitstream priority partitioning can be used. Here, the video bitstream can be partitioned into segments of different priorities according to their relative impact on the video quality. These priority rankings can then be used in an unequal error protection fashion at the physical layer that increases the robustness of the video sequence [6,7]. At the physical layer, UEP is achieved by using Forward Error Correction codes and hierarchical modulation where each mapped symbol is represented by the Most Significant Bit (MSB) and the Least Significant Bit (LSB), thus creating unequal error protection at bit level [8-12].

In this paper we propose a novel UEP scheme to transmit H.264/AVC data over wireless Rayleigh channel employing Bose-Chauduri-Hocquengem (BCH) code as a FEC and non-uniform 8PSK modulation. Simulation results show that compared with non-hierarchical methods, the proposed UEP approach provides more effective video transmission through noisy wireless channels.

The remainder of this paper is organized as follows. Section 2 briefly introduces the H.264/AVC standard along with a packet priority assignment schemes. Section 3 outlines the coding concepts associated with the $\mathrm{BCH}$ code as well as the non-uniform 8-PSK modulation in UEP. In Section 4, we describe the processes involved in the design and implementation associated with the proposed scheme. Section 5 explains the experimental set-up and presents the simulation results as well as discussions for the 1/2-rate $\mathrm{BCH}$ code designs with non-uniform 8 PSK modulation applied to the prioritized H.264/AVC video data. Finally, Section 6 concludes the paper. 


\section{H.264/Ave Partitioning And Priotitization}

The H.264/AVC video coding standard [1,2] proposed by the Joint Video Team (JVT) of ITU-T and ISO/IEC, achieves a significant improvement in the compression efficiency over the other existing standards. This makes H.264 AVC a favorable candidate for all future multimedia applications.

The H.264/AVC standard is composed of two layers namely the Video Coding Layer (VCL) for video compression and Network Abstraction Layer (NAL) for transport of compressed video data over networks. NAL works in two kinds of mode: Single Slice mode and Data Partition mode. When using Data Partition mode, H.264 puts all variable length codes with the same data type together in each frame.

Header information includes head information, macroblock type, frame type, predicted residual of motion vectors, frame flag and this part is called Data Partition A (DP A), which is the most important part. Intra-frame segmentation is called Data Partition (DP B). It loads the coding mode and the correlation coefficient in intra frame blocks. Compared with information of inter-frame data partitions, intra-frame information can prevent further drift, thus it is more effective than inter-frame data partitions. Inter-frame segmentation is called Data Partition C (DP C). It only includes the coding mode and the correlation coefficient in inter-frame block; it is the biggest segmentation in the video stream. Without receiving the DPA data of a slice correctly, DP B and DP C packets cannot be decoded, thereby discarding the whole slice data. Whereas if a DP C is lost, then the slice can be decoded using the received DP A and DP B information [1,3].

The output of the H.264/AVC source can be organized in packets of varying importance, depending on the frame type, DP type and slice group. Therefore we can exploit the unequal importance of video data at the application layer by packetizing all the important data together, prioritizing them accordingly and give more protection to them. The H.264/AVC bitstream is split into three priorities, with the Data partitioning mode ' $O N$ ' and the data of each GOP is differentiated based on the frame type and DPs. In order to exploit unequal error protection at the application layer the three priority queues have been designed as follows [3]:

\section{Priority 1 Queue}

IDR - frame

DPA of I- frame

DPB of I- frame (if queue 1 can accommodate else move data to queue 2)

Priority 2 Queue

DPA of P- frame

DPA of B- frame

DPC of P- frame (if queue 2 can accommodate else move data to queue 3 )

Priority 3 Quene

DPC of P- frame

DPB of B- frame

DPC of B- frame

\section{Bch Code And Non-Uniform 8psk Modulation}

The $\mathrm{BCH}$ code is an amazing generalization of the Hamming code [13] for more than one error detection and correction. The $\mathrm{BCH}$ codes have been subject to lots of attention and studies since it was invented. The $\mathrm{BCH}$ code allows the user to control the block length and the acceptable number of errors. Therefore the $\mathrm{BCH}$ code can be custom built for given specification [13].

The complexity of decoding convolutional codes (viterbi) increases exponentially as the constraint length increases [13]. The main advantage using the $\mathrm{BCH}$ code is the decoding process called the syndrome decoding algorithm [13]. The syndrome decoding is implemented using simple electronic devices and can easily be done without the help of PC's. The decoding device can be made very compact and operating on low power batteries. For any positive integers $m(m \geq 3)$ and $t(t<2 m-1)$, there exists a binary $\mathrm{BCH}$ code with the following parameters:

$$
\begin{array}{ll}
\text { The block length: } & N=2^{m}-1 \\
\text { Number of parity check: } & N-K \leq m t \\
\text { Minimum distance: } & d_{\min } \geq 2 t+1
\end{array}
$$

The $\mathrm{BCH}$ code can correct up to $t$ errors and is commonly called as $t$-errors-correcting $\mathrm{BCH}$ code. The encoding of such a code begins by defining a Galois field. The Galois field is also called the finite field. A Galois field containing $\mathrm{q}$ elements is denoted as $\mathrm{GF}(\mathrm{q})$. The generator polynomial $\mathrm{g}(\mathrm{X})$ has the following roots: $\alpha, \alpha^{2}, \alpha^{3}, \ldots$, $\alpha^{2 t}$. Also its conjugates are also roots.

The non-zero elements of the $\operatorname{GF}\left(2^{\mathrm{m}}\right)$ are roots of $\mathrm{X}^{2^{\wedge} \mathrm{m}-1}+1$ and all the roots of $\mathrm{g}(\mathrm{X})$ are elements of $\mathrm{GF}\left(2^{\mathrm{m}}\right)$. Therefore it can be said that $\mathrm{g}(\mathrm{X})$ divides $\mathrm{X}^{2 \wedge \mathrm{m}-1}+1\left(\mathrm{~g}(\mathrm{X})\right.$ is a factor of $\left.\mathrm{X}^{2^{\wedge} \mathrm{m}-1}+1\right)$ this can be used to generate a cyclic code of length $2^{\mathrm{m}}-1$.

Therefore the generator polynomial for a $t$-error-correcting binary $\mathrm{BCH}$ code of length $2^{m}-1$ is given by 


$$
\mathrm{g}(X)=\operatorname{LCM}\left\{\phi_{1}(X), \phi_{2}(X), \ldots, \phi_{2 t}(X)\right\}
$$

For $1 \leq \mathrm{i} \leq 2 t$, let $\Phi_{\mathrm{i}}$ be the minimal polynomial of $a_{i}$

If $i$ is an even integer, it can be termed as $\mathrm{i}^{\prime} \mathrm{i}^{\prime} 2^{\mathrm{L}}$, where $L$ is a positive integer. Then $a_{i}$ is a conjugate of $a_{I}{ }_{I}$ and hence they have the same minimal polynomial.

$$
\phi_{i}(X)=\phi_{i^{\prime}}(X)
$$

Every even power of a is a sequence of $\alpha, \alpha^{2}, \alpha^{3}, \ldots, \alpha^{2 t}$ and has the same minimal polynomial as odd power $\alpha$ in the sequence Therefore the generator polynomial can be reduced to:

$$
\mathbf{g}(X)=\operatorname{LCM}\left\{\phi_{1}(X), \phi_{3}(X), \ldots, \phi_{2 t-1}(X)\right\}
$$

There are only t minimal polynomials in the above equation, the degree in $\mathrm{GF}\left(2^{\mathrm{m}}\right)$ is at most $\mathrm{m}$ and the degree of $\mathrm{g}(\mathrm{X})$ is at most $\mathrm{mt}$.

Therefore the $t$-error-correcting binary $\mathrm{BCH}$ code generated by $\mathrm{g}(\mathrm{X})$ has at most $\mathrm{mt}$ parity check bits and its dimension at least $2^{m}-m t-1$. The $\mathrm{BCH}$ codes generated are called as primitive or narrow sense $\mathrm{BCH}$ codes since they are constructed from primitive elements a in $\mathrm{GF}\left(2^{\mathrm{m}}\right)$.

The $\mathrm{BCH}$ decoding procedure is simple and fast and can be done in the following steps [13]:

1. Compute the syndrome $S=\left(S_{1}, S_{2}, \ldots, S_{2 t}\right)$ of the received polynomial $r(X)$.

2. Determine the error location polynomial $\sigma(X)$ from the Newton identities.

3. Determine the roots $\beta_{1}^{-1}, \beta_{2}^{-1}, \ldots, \beta_{\nu}^{-1}$ of $\sigma(X)$ and take the inverses of these roots to obtain the error location numbers $\beta_{1}=\alpha^{\jmath_{1}}, \beta_{2}=\alpha^{\jmath_{2}}, \ldots, \beta_{\nu}=\alpha^{j_{\nu}}$.

4. Form the error pattern

$$
\mathbf{e}(X)=X^{j_{1}}+X^{j_{2}}+\cdots+X^{j_{\nu}}
$$

5. Error correction is performed by adding(modulo-2) $e(X)$ to $r(X)$ to give the code polynomial $\mathrm{v}(\mathrm{X})=\mathrm{r}(\mathrm{X})+\mathrm{e}(\mathrm{X})$.

\section{Non-Uniform 8-PSK modulation}

Non-Hierarchical or uniform 8 Phase Shift Keying (PSK) uses 8 different possible phases to send 3 bits per symbol. The data rate is therefore doubled compared to the QPSK for the same bandwidth.

Non uniform 8PSK consists of mapping the 8 constellation points at different angles rather than uniform angle separation between points $[14,15]$. The angle between the points can be represented as:

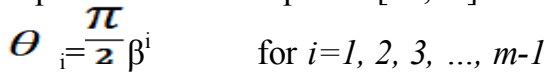

$m$ : number of bits used for one symbol

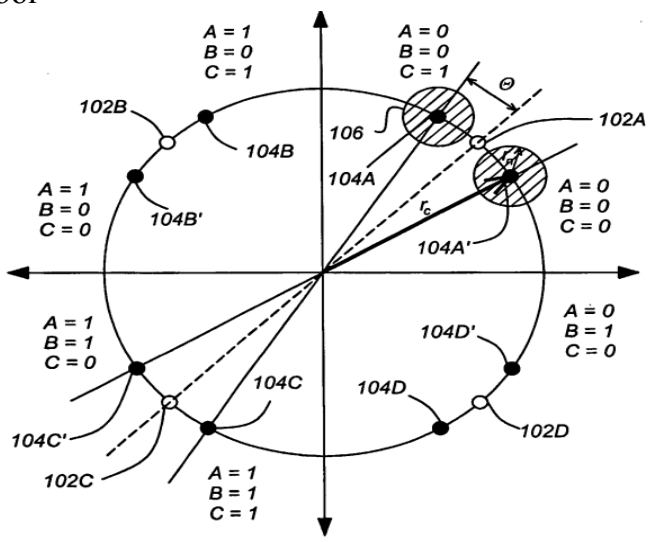

Fig. 1: Non-Uniform 8PSK modulation constellation.

Fig. 1 shows how the points are represented on the constellation diagram. The phase angles vary according to the Beta $(\beta)$. The reason for fixing the ratio of the angles for any subsequent levels of the signal constellation hierarchy $\beta$ to a constant is to facilitate the phase offset optimization (i.e., only a single parameter needs to be optimized rather than manipulating $(m-1)$ variables[15]). Non uniform 8PSK has shown better performance than 
uniform PSK. When Beta $(\beta)=0.5$, they constellation becomes similar to a uniform 8PSK constellation. Also, the modulator is designed and implemented based on the following mapping rules:

$$
\begin{aligned}
& \text { Phase Angle } \\
& \left(\frac{\beta \pi}{2}\right)+\left(\frac{\beta^{2} \pi}{2}\right) \quad S_{0} \quad 000 \\
& \left(\frac{\beta \pi}{2}\right)-\left(\frac{\beta^{2} \pi}{2}\right) \quad S_{1} \quad 001 \\
& 2 \pi-\left[\left(\frac{\beta \pi}{2}\right)+\left(\frac{\beta^{2} \pi}{2}\right)\right] \quad S_{2} \quad 010 \\
& 2 \pi-\left[\left(\frac{\beta \pi}{2}\right)-\left(\frac{\beta^{2} \pi}{2}\right)\right] S_{3} \quad 011 \\
& \pi-\left[\left(\frac{\beta \pi}{2}\right)+\left(\frac{\beta^{2} \pi}{2}\right)\right] S_{4} \quad 100 \\
& \pi-\left[\left(\frac{\beta \pi}{2}\right)-\left(\frac{\beta^{2} \pi}{2}\right)\right] \quad S_{5} \quad 101 \\
& \pi+\left[\left(\frac{\beta \pi}{2}\right)+\left(\frac{\beta^{2} \pi}{2}\right)\right] \quad S_{6} \quad 110 \\
& \pi-\left[\left(\frac{\beta \pi}{2}\right)-\left(\frac{\beta^{2} \pi}{2}\right)\right] \quad S_{7} \quad 111
\end{aligned}
$$

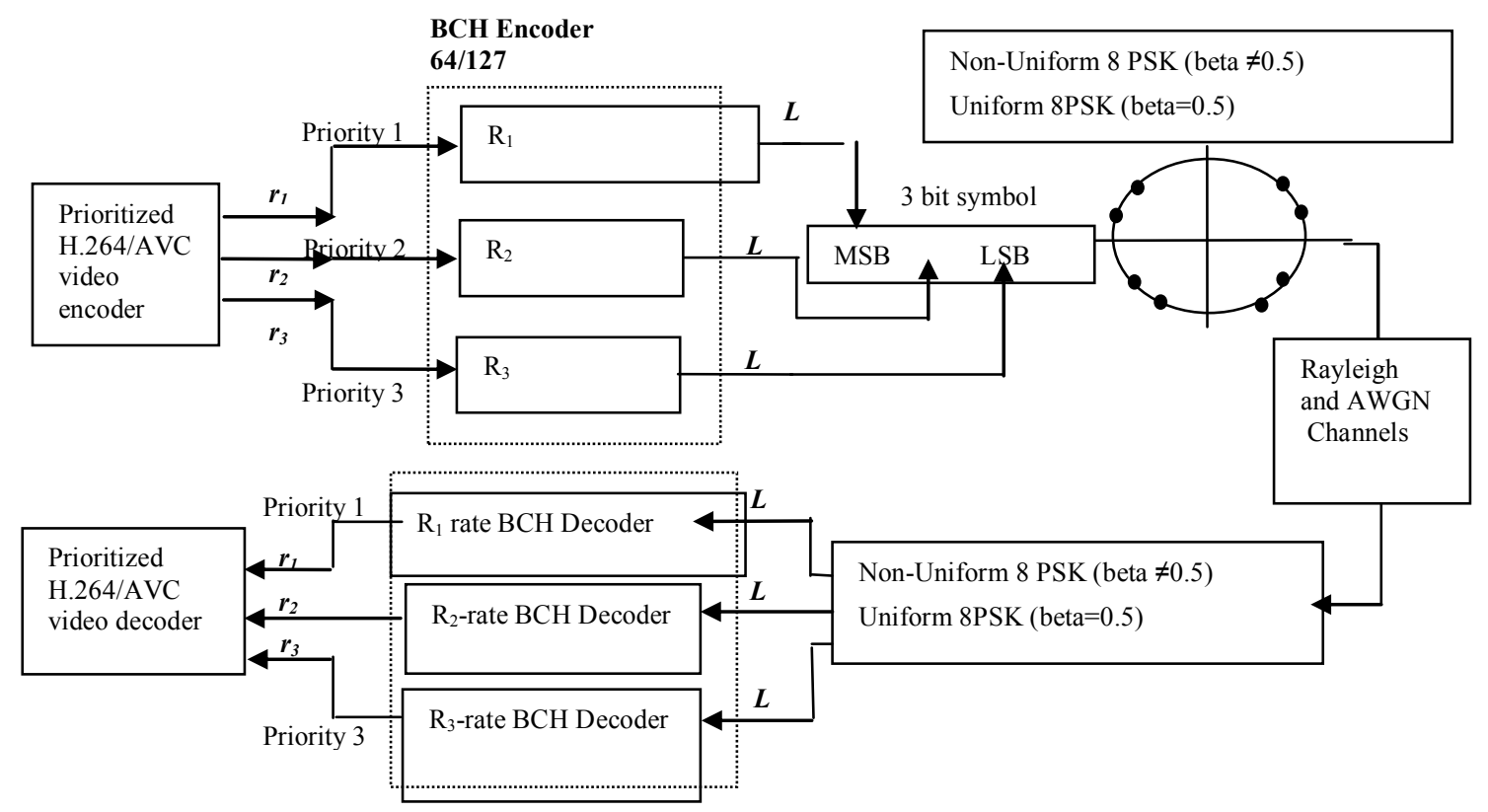

Fig. 2: Block diagram of the proposed UEP system

\section{Proposed System Set-Up And Design}

The design of the proposed cross-layer UEP scheme, employing prioritized H.264/AVC video data at the application layer and equal-rate as well as prioritized $\mathrm{BCH}$ codes with non-uniform 8-PSK modulation at the physical layer, are explained in this section. Fig. 2 shows the block diagram of our robust transmission scheme which uses equal-rate $\mathrm{BCH}$ encoders and non-uniform 8PSK modulation. Fig. 2 shows the scheme in the form of a block diagram. We apply the equal rate $B C H$ code $\left(R_{1}=R_{2}=R_{3}\right)$ to packets of each priority queue of H.264 video output. The outputs of the three codes are then multiplexed to form non-uniform 8PSK symbols by using 1 bit from each of the three priority queues to form a symbol.

Here, the data from the highest (lowest) priority queue is assigned to the MSB (LSB) bit of the symbol, the middle priority data being assigned to the middle bit of the symbol. For a $512 \mathrm{kbps}$ video data, the length of 
each queue would be $r_{1}=r_{2}=r_{3}=512 / 3 \mathrm{kbps}$. By applying a $64 / 127 \mathrm{BCH}$ code to every queue (i.e., $R_{1}=R_{2}=R_{3}=$ $1 / 2$ ), the output data rate would be $1024 \mathrm{kbps}$ and hence an overall $1 / 2$ rate physical layer UEP transmission scheme is achieved. The modulated symbol is sent over the wireless Rayleigh channel and the received bit stream is demodulated using hard-decision non-uniform 8-PSK demodulation. The bitstream is then demultiplexed and fed to the corresponding $\mathrm{BCH}$ decoder and the queues are arranged in order of their priorities and inputted to the H.264 decoder. As H.264/AVC is not designed to handle bit errors, any bit errors will lead to discarding of the packet. The bitstream with packet drops is given to the robust H.264/AVC decoder which reconstructs the video frames with the help of error concealment techniques.

\section{Simulation Results And Discussions}

The experiment is set up by using the H.264/AVC Annex B (JM14.1) reference source code [1] for encoding the CIF (352x288 pixels) Bus video test sequence at a bitrate of $512 \mathrm{Kbps}$. The video sequence was encoded at 30 frames/second for a GOP of 12 frames (IDR B P B P B P B P B P B I ...) with IDR rate of 24 frames. The following error resiliency features was enabled: data partitioning, FMO dispersed mode and Constrained Intra Prediction. The NAL size of 200 bytes was considered. A robust decoder with error concealment schemes is used [3].

Two channel models namely Rayleigh and AWGN have been used in the simulations. The built in AWGN and Rayleigh MATLAB R2013a Simulink channel models, from the communications blockset, were used. The experiments were conducted by varying the Signal to Noise Ratio per bit (Eb/No) from -4 to $20 \mathrm{~dB}$ in steps of $2 \mathrm{~dB}$ and by varying asymmetric 8-PSK modulation design parameter $\beta$ from 0.30 to 0.50 in steps of 0.05. For simulations Visual studio 2010 is used to compile H.264/AVC source code, MATLAB R2013a Simulink to simulate the physical layer and Cygwin to automate the complete system.

Figs. 3 to 5 presents the BER performances for the three levels of protection. It can be clearly seen that while the modulation parameter beta is varied UEP is perfectly achieved by our new design. At beta $=0.30$ and a BER of $10^{-4}$ we show that the highest prioritized queue yields a gain of around $6 \mathrm{~dB}$ over the medium priority data. From the simulation results of Fig. 5 it can be clearly observed that for all the designed UEP schemes and for the Highest prioritized queue, as beta increases from 0.30 to 0.50 the error rates reduces, hence both the $\mathrm{BCH}$ code as well as the non-uniform 8PSK modulator have more UEP capabilities than the uniform case.

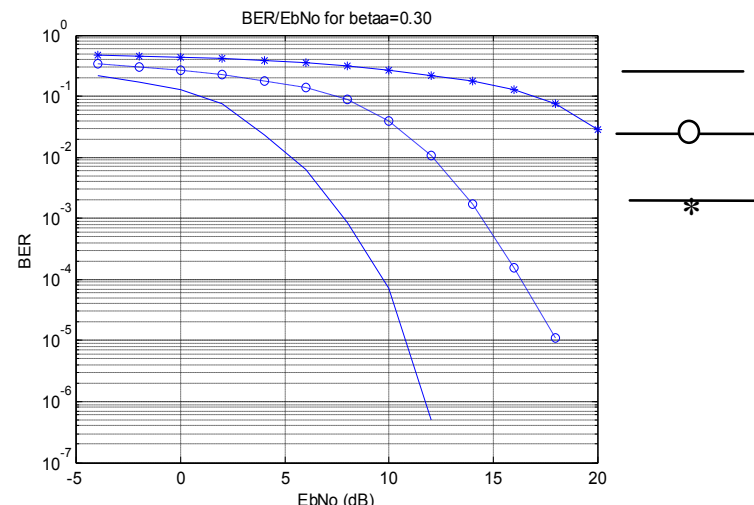

High Priority

Medium Priority

Low Priority

Fig. 3: Bit error performance at beta $=0.30$

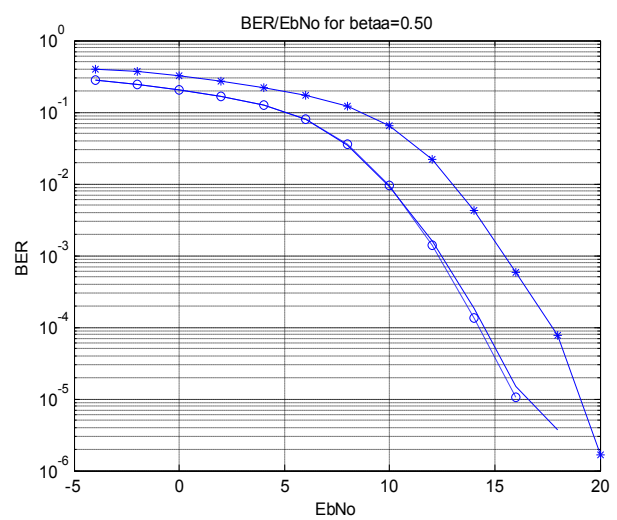

Fig. 4: Bit error performance at beta $=0.50$ 
Figs. 6 -9 show the test video pictures saved for various UEP schemes that have been designed and implemented in this research work. It can be clearly observed that all UEP schemes employing $1 / 2$-rate BCH codes and hierarchical modulation outperform the non-hierarchical 8PSK modulation case. The frame number at which the best performance occur for all schemes together with the PSNR, Eb/No as well as beta values used in the simulations are also given.

\section{Conclusions}

The H.264/AVC video compression standard represents the state-of-the-art in compression techniques. In addition to achieving a high compression rate, H.264 offers several error resilience tools to detect, localize and conceal errors in the compressed bitstream. However, these tools can only work with a limited number of errors. Since wireless channels can have high BERs, channel coding coupled with hierarchical modulation are needed to correct errors in the bitstream. H.264/AVC compressed video is well-suited for unequal error protection to ensure the important parts of the video are received with a minimum number of errors. We designed and implemented a novel unequal error protection scheme employing $\mathrm{BCH}$ channel coders and Hierarchical modulators/demodulators and performed experiments transmitting the H.264/AVC bitstreams through a simulated wireless Rayleigh fading channel with varying amounts of errors. The results of these experiments have shown the advantage of using unequal error protection for protecting H.264/AVC simple encoded video data. By exploiting the prioritized structure of the source coded bitstream, unequal error protection is able to obtain the high quality reconstructed video for a source bitrate of $512 \mathrm{Kbps}$.

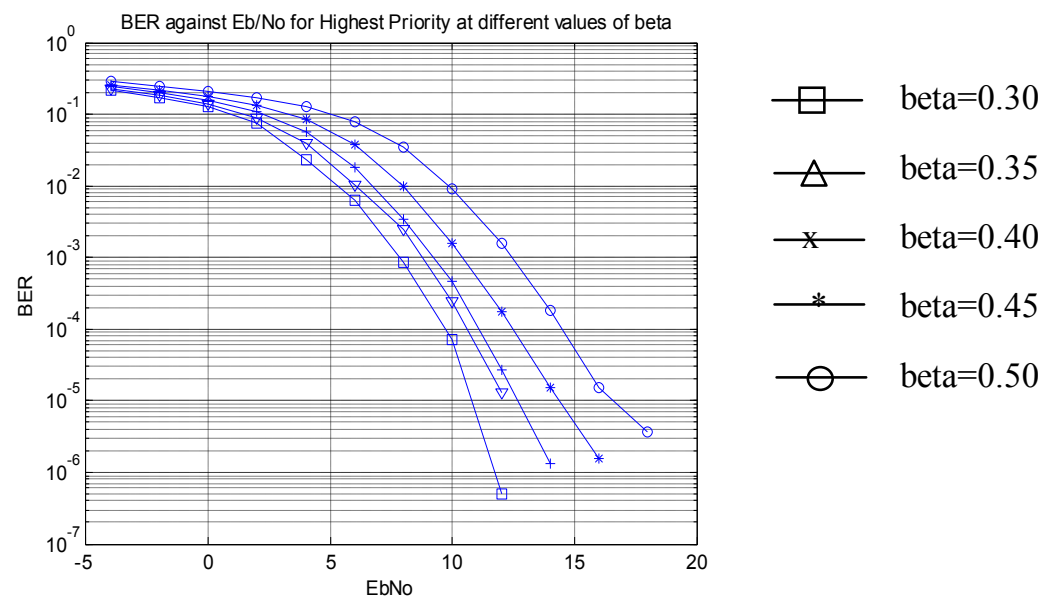

Fig. 5: Bit error performance for the Highest priority at different values of beta.

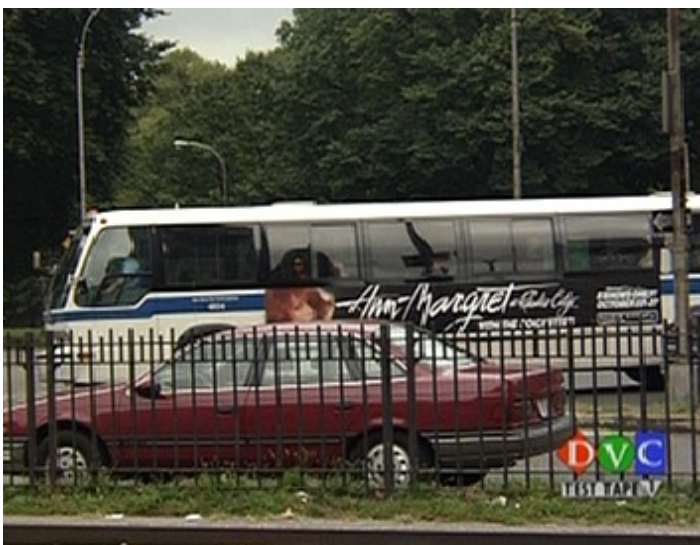

Fig. 6: Original video sequence, $P S N R=28.2 \mathrm{~dB}$ $25 \mathrm{~dB}$

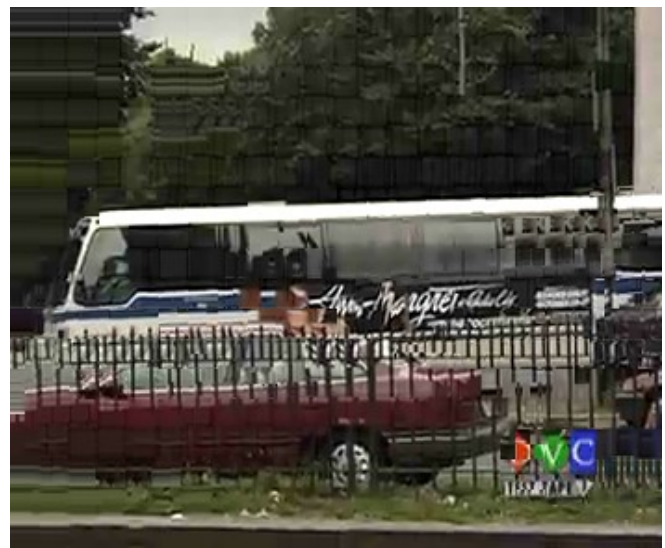

Fig. 7: Video sequence at $E_{b} N_{o}=14 d B, P S N R=$ beta $=0.30$ 


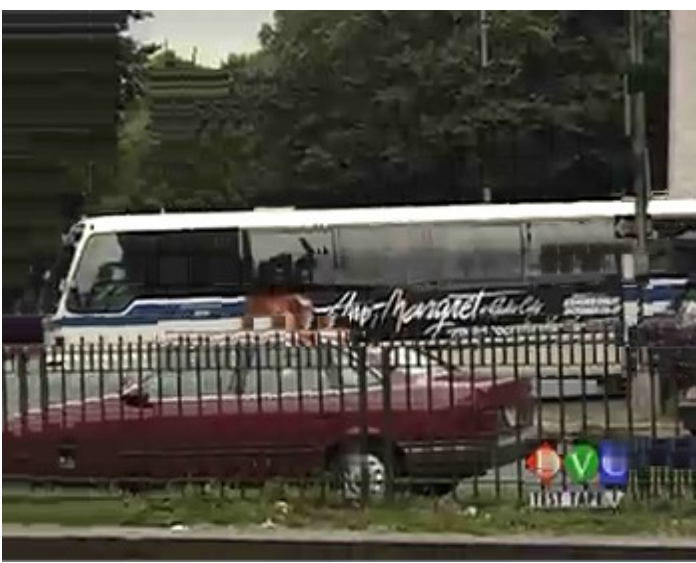

Fig. 8: Video sequence at $\mathrm{E}_{\mathrm{b}} \mathrm{N}_{\mathrm{o}}=14 \mathrm{~dB}, \mathrm{PSNR}=26 \mathrm{~dB}$ $22 \mathrm{~dB}$ beta $=0.40$

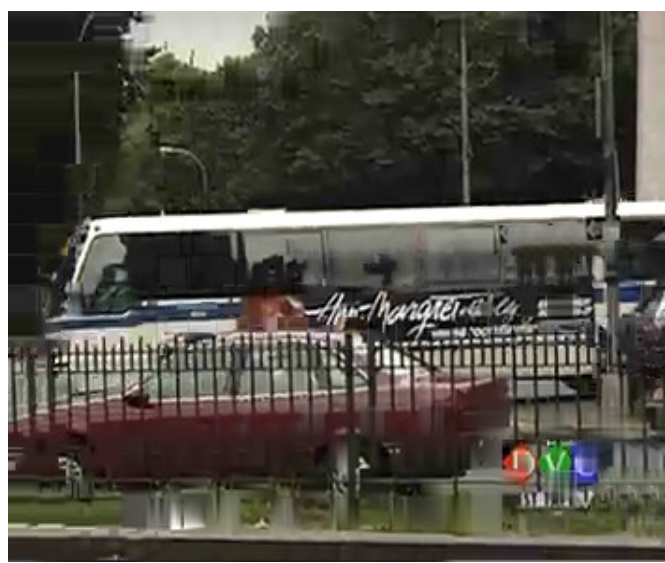

Fig. 9: Video sequence at $\mathrm{E}_{\mathrm{b}} \mathrm{N}_{\mathrm{o}}=14 \mathrm{~dB}, \mathrm{PSNR}=$ beta $=0.50$

\section{References}

[1]. "Draft ITU-T recommendation and final draft international standard of joint video specification (ITU-T Rec. H.264/ISO/IEC 14 496-10 AVC," in Joint Video Team (JVT) of ISO/IEC MPEG and ITU-T VCEG, JVTG050r1, 2003.

[2]. T. Wiegand, G. J. Sullivan, G. Bjøntegaard, and A. Luthra, "Overview of the H.264/AVC video coding standard," IEEE Trans. Cir. Syst. Video Technol., Vol. 13, pp. 560-576, July 2003

[3]. S. Kumar, L. Xu, M. K. Mandal, and S. Panchanathan, "Error resiliency schemes in H.264/AVC video coding standard," Elsevier J.Visual Communication and Image Representation (Special issue: Emerging H.264/AVC Video Coding Standard), Vol. 17(2), pp. 425-450, April 2006

[4]. L. F. Wei, "Coded modulation with unequal error protection," IEEE Trans. Commun., Vol. 41(10), pp. 1439-1449, Oct. 1993.

[5]. A. R. Calderbank and N. Seshadri, "Multilevel codes for unequal error protection," IEEE Trans. Inf. Theory, Vol. 39(7), pp. 12341248, July 1993.

[6]. W. H. Heinzelman and R. Talluri, "Unequal error protection of MPEG-4 compressed video," Proc. International Conf. Image Processing, Vol. 2, pp. 530-534, 1999.

[7]. A. Bhatnagar, S. Kumar, A. Janarthanan, A. Annamalai Jr, M. Medley and J. Matyjas, “A multilevel unequal protection scheme for robust H.264/AVC video transmission over wireless channels," IEEE MILCOM, Boston, MA, USA, Oct. 2009.

[8] B.Masnick, J.Wolf, " On Linear Unequal Error Protection Codes", IEEE Transactions on Information Theory, Vol-It 3, No. 4, pp600-607, Oct. 1967.

[9] J. van Gils, "Two topics on linear unequal error protection codes: bounds on their length and cyclic code classes", IEEE Trans. Inform. Theory, Vol. 29 No. 2, pp. 866-876, 1983.

[10]. M. B. Pursley and J. M. Shea, "Non-uniform phase-shift-key modulation for multimedia multicast transmission in mobile wireless networks," IEEE J. Sel. Areas. Commun., Vol. 17(5), pp. 774-783, May 1999.

[11]. M. B. Pursley and J. M. Shea, "Adaptive non-uniform phase-shift-key modulation for multimedia traffic in wireless networks," IEEE J. Sel. Areas. Commun., Vol. 18(6), pp. 1394-1407, Aug. 2000.

[12]. M. B. Pursley and J. M. Shea, "Multimedia multicast wireless communications with phase-shift-key modulation and convolutional coding," IEEE J. Sel. Areas. Commun., Vol. 17( 9), pp. 1999-2010, Nov. 1999.

[13] S. Lin, D. Costello, "Error Control Coding: Fundamentals and Applications" Prentice-Hall, Englewood Cliffs, N.J, USA, 1983.

[14] P.Vitthaladevuni and M. Alouini, "Exact BER computation of generalized hierarchical PSK constellations," IEEE Transactions on Communications, Vol. 51, pp. 2030-2037, Dec. 2003

[15] J. Lu, A. Annamalai Jr., and D. R. Vaman, "Reducing Signal Distortion Due to Transmission Error via Multi-resolution Digital Modulations", Wireless Telecommunications Symposium, April 2008, pp 360-369.

[16] M. Sajadieh, F. R. Kschischang, and A. Leon-Garcia, "Modulation-assisted unequal error protection over the fading channel," IEEE Trans. Veh. Technol., vol. 47, no. 5, pp. 900-908, Sep. 1998

[17] M. Isaka and H. Imai, "Hierarchical coding based on adaptive multilevel bit-interleaved channels," in Proc. Veh. Technol. Conf., Spring 2000, pp. 2227-2231. 